\title{
Single-Molecule AFM Studies of Substrate Transport by Using the Sodium-Glucose Cotransporter SGLT1
}

\author{
Theeraporn PunTheERANuRAK* \\ Department of Biology, Faculty of Science, Mahidol University and NANOTEC \\ Center of Excellence at Mahidol University, Bangkok, 10400, Thailand \\ Rolf K. H. Kinne \\ Department of Epithelial Cell Physiology, Max Planck Institute of Molecular Physiology, Dortmund, 44227, Germany \\ Hermann J. Gruber and Peter Hinterdorfer \\ Institute for Biophysics, Johannes Kepler University of Linz, Linz, A-4040, Austria
}

(Received 15 July 2007)

\begin{abstract}
In an apical membrane of epithelial cells from the small intestine and the kidney, the high-affinity $\mathrm{Na}^{+}$/D-glucose cotransporter type 1 (SGLT1) plays a crucial role in intestinal glucose absorption and in renal glucose reabsorption. Here, the over-expression of rabbit SGLT1 in rbSGLT1transfected Chinese hamster ovary $(\mathrm{CHO})$ cells was first characterized using the immuno-staining method on non-permeabilized cells. The cells were then imaged with atomic force microscopy (AFM), revealing live and fixed cells strongly attached to the glass surfaces. A bioconjugate chemistry approach was employed to functionalize the surfaces of the AFM tips with D-glucose molecules via three different heterobifunctional crosslinkers. The D-glucose binding site and the translocation pathway of SGLT1 were investigated by studying the interaction forces between tip-bound D-glucose and SGLT1 in live cells on the single-molecule level. Analysis of these forces suggested that a long crosslinker with a small end group might be suitable for probing the D-glucose transport pathway of SGLT1. We show that single-molecule AFM technology is a powerful method for investigating transmembrane proteins and transporter functions in live cells.
\end{abstract}

PACS numbers: 07.79.Lh, 82.37.Rs, 87.15.Kg, 87.83.+a

Keywords: SGLT1, Na+-D-glucose cotransporter, AFM, Force spectroscopy

\section{INTRODUCTION}

Molecular recognition in biological systems between receptors/ligands, proteins/antibodies and substrate/ transporter is often the initiating step in a wide variety of biological functions. A Na ${ }^{+}$-D-glucose cotransporter type 1 (SGLT1) is one of the most intensively studied membrane transporters [1,2]. SGLT1 transports glucose into cells driven by the $\mathrm{Na}^{+}$electrochemical potential difference across the membrane [3]. Recently, our group has pioneered an investigation of the topology and the substrate binding of SGT1 on living cells by using atomic force microscopy (AFM) [4]. The principle and the operation of the AFM have been described extensively [5-7]. This approach is nowadays widely used because the experiments can be performed on unaltered cells under near-physiological conditions without modifications of the membrane proteins $[8,9]$. Furthermore, the

\footnotetext{
*E-mail: sctpt@mahidol.ac.th; Fax: +66-2-354-7161
}

high sensitivity of the AFM and the soft cantilevers allow for the detection of $\mathrm{pN}$ forces of transporter-ligand complexes, providing a unique opportunity to detect molecular recognition of single molecular binding events under different experimental conditions [4,8-14].

In the current study, we extended the molecular recognition force spectroscopy of the glucose-SGLT1 interaction by using novel crosslinkers for the coupling of Dglucose to AFM tips. Our studies demonstrate that a newly developed acrylamide crosslinker might be a powerful candidate for a detailed investigation of the sugar translocation pathway of SGLT1.

\section{EXPERIMENTS}

1. Characterization of SGLT1 in the G6D3 Cells: Fluorescent Immuno-staining

Freshly isolated G6D3 cells (CHO cells stably transfected with rbSGLT1) at passages below fifteen were 


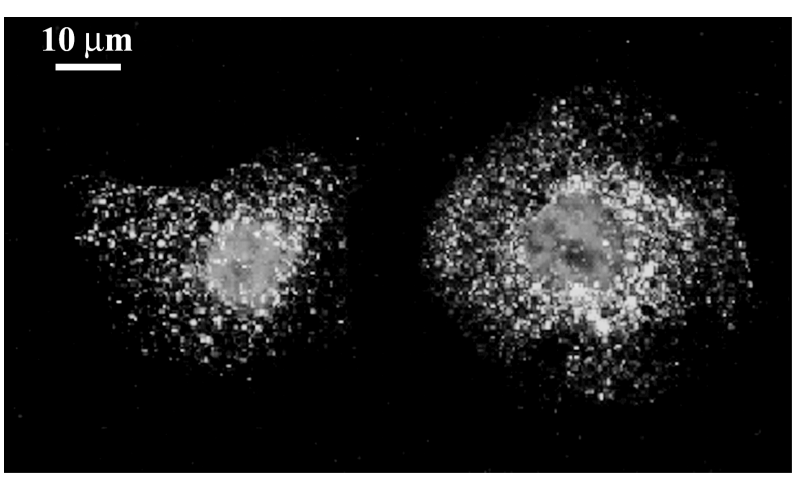

Fig. 1. Detection of SGLT1 on the surface of nonpermeabilized G6D3 cells fixed with paraformaldehyde. Specific QIS30 antibodies were bound to SGLT1 on cell membranes and subsequently stained by incubation with antirabbit IgG linked to $\mathrm{Cy} 3$ fluorophor (spreading white color). Nuclei are shown in the middle of the cells (DAPI staining). The scale bar represents $10 \mu \mathrm{m}$.

used for all experiments. In order to confirm the over-expression of SGLT1 on the surface of the G6D3 cells used in this study, we employed the fluorescent immuno-staining method. The G6D3 cells forming a uniform monolayer on poly-L-lysine-coated glass cover-slips were fixed with paraformaldehyde. For surface labeling, QIS30 antibody (specific antibody against loop 6-7 of SGLT1 [15]) was used on non-permeabilized cells, followed by incubation with anti-rabbit IgG linked to Cy3 fluorophor. Nuclei were stained with 4'-6-diamidino-2phenylindole (DAPI). As illustrated in Figure 1, SGLT1 was clearly detected on the cell surface by using epifluorescence microscopy. The incubation of the specimens lacking the primary antibody revealed no observable fluorescence (data not shown).

\section{AFM Imaging of G6D3 Cells}

The AFM offers the potential for imaging the morphology of living cells in a non-destructive manner. Monolayers of live G6D3 cells grown on poly-L-lysine-coated glass cover-slips were imaged at room temperature in a KRH-Na ${ }^{+}$medium. A contact mode AFM (PicoSPM II, Molecular Imaging, Tempe, AZ) at forces of less than 100 $\mathrm{pN}$ with non-functionalized AFM cantilevers (cantilever from Veeco $\mathrm{GmbH}$, Mannheim, Germany) of 0.03 to 0.5 $\mathrm{N} / \mathrm{m}$ nominal spring constants were utilized. Large-scale topography and deflection images of the living cells are shown in Figures 2(a) and 2(b), respectively. The region surrounding the nucleus appeared flat and assisted adhesion of the cells to the glass plate. Typical cells were about $30-70 \mu \mathrm{m}$ in diameter, with a characteristic bright zone corresponding to the nuclei region appearing to be about $1-3 \mu \mathrm{m}$ in height. Due to the high flexibility and the softness of the cell membranes of living cells, we were not able to achieve a higher resolution on
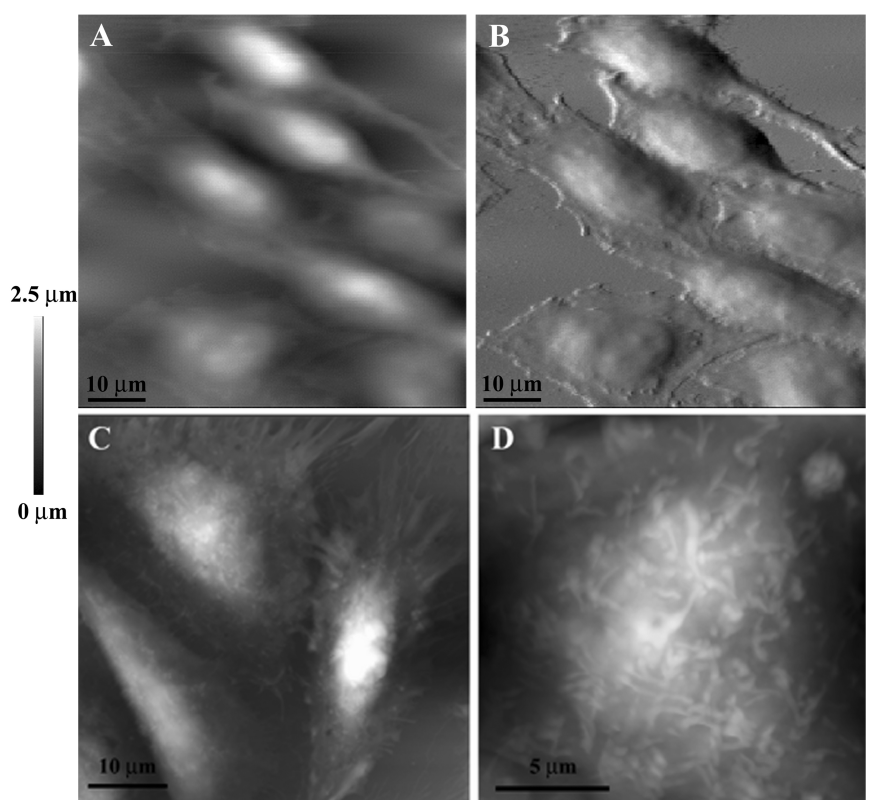

Fig. 2. AFM images of living and fixed G6D3 cells: (a) Topographical image and (b) deflection image of living cells imaged in KRH-Na medium at a scan size of $80 \mu \mathrm{m}$ by using a poly-L-lysine-coated glass cover-slip. The bright regions of the images correspond to the area of the nucleus. (c) Topographical image of fixed cells acquired at a scan size of 50 $\mu \mathrm{m}$. (d) Low-scan size image $(17 \mu \mathrm{m})$ of the cell surface of fixed cells. The images show the substructures of the cell membrane, which are most likely microvilli.

the membrane surface. The surface morphology, such as lamellipodia and microvilli, could only be observed after cell fixation, as illustrated in Figures 2(c) and 2(d). Nevertheless, the AFM images revealed that the G6D3 cells were strongly attached to the surface, which was a prerequisite for atomic force microscopy. We achieved about an $80 \%$ confluent-cell monolayer, which contained cells with a maximum height of about $1 \mu \mathrm{m}$. The G6D3 cells were easily grown and could be maintained alive at room temperature for at least six hours, which was suitable for AFM force spectroscopy.

3. Single-molecule Recognition of D-glucose Coupled via Three Kinds of Crosslinkers

Recently, we have demonstrated that AFM force spectroscopy is a powerful method to probe the substratetransporter interaction of SGLT1 in a live cell system [4]. We established a new method for the investigation of the membrane topology and the initial sugar-binding site of SGLT1. However, due to the bulky group of AFM crosslinkers, we were only able to detect the interactions on the surfaces of the cells. Here, a new crosslinker with a smaller end group, i.e., acrylamide (AM) and a longer polymer length was used for comparison with our standard vinylsulfon (VS) crosslinker, which was shown to 


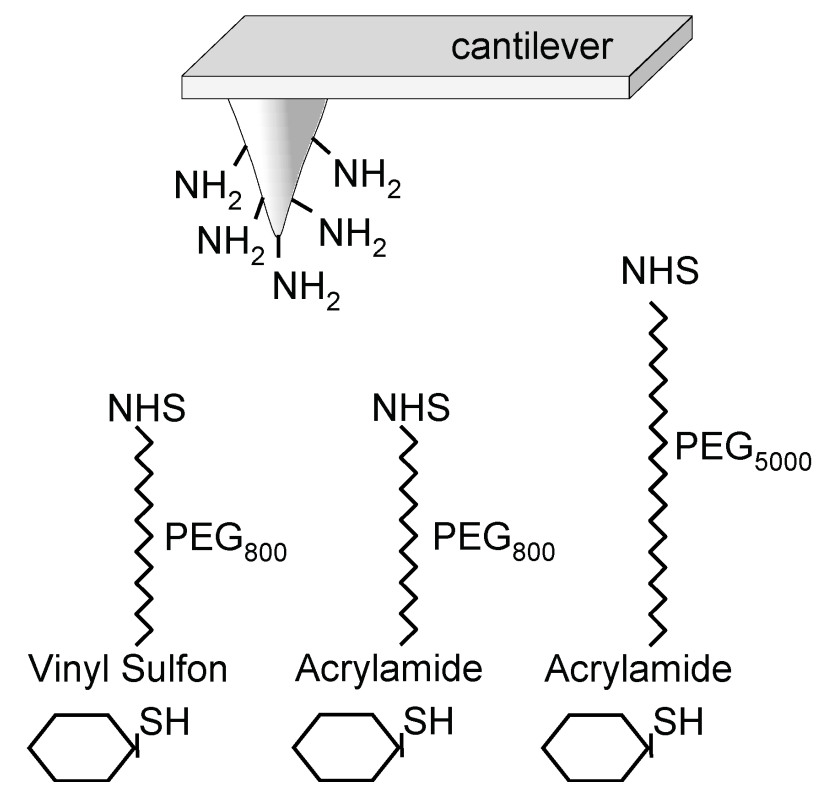

Fig. 3. Linkage of D-glucose to AFM tips. Sugars were covalently coupled to AFM tips via heterobifunctional PEG derivatives.

be apt for examining the initial sugar-binding site of SGLT1 [4]. We coupled 1-thio- $\beta$-D-glucose to AFM tips through three different flexible PEG [poly(ethylene glycol)] crosslinkers at a very dilute surface density. In brief, silicon tips were first functionalized with ethanolamine and the NHS (N-hydroxysuccinimide) end of the PEG linkers (NHS-PEG 800 -VS, NHS-PEG 800 -AM and NHS$\left.\mathrm{PEG}_{5000}-\mathrm{AM}\right)$ was covalently bound to amines on the tip surface, followed by the attachment of sugar via a free SH group (Figure 3).

For the glucose/SGLT1 recognition, force-distance cycles in $\mathrm{KRH}-\mathrm{Na}^{+}$buffer were performed by using ligandcoated cantilevers (rectangular, Veeco $\mathrm{GmbH}$ ) with $0.02-\mathrm{N} / \mathrm{m}$ nominal spring constants in the conventional contact-force-calibration-plot mode. Force-distance cycles were recorded on cell surfaces with the assistance of a CCD camera for the localization of the AFM cantilever on isolated cells or cell monolayers. The principle of single-molecule-recognition force detection of the sugar-transporter complex on living G6D3 cells by using AFM is illustrated in Figure 4(a). In the force-distance mode, the deflection angle of the cantilever is measured as a function of the vertical position of the cantilever. At a fixed lateral position, the tip first approached the surface of the cells (dotted trace). Far away from the surface (1), no bending of the cantilever is visible and the cantilever deflection $(\Delta z)$ and thus, the force $(f)$ are almost zero (the force is calculated according to Hook's law; i.e., $f=k \Delta z$, where $k$ is the spring constant of the cantilever). As the cantilever reaches the cell surface (2), the cantilever is deflected upwards due to the repulsive tip-sample interaction. Subsequently, the tip is retracted (retrace) and relaxation of the repulsive force
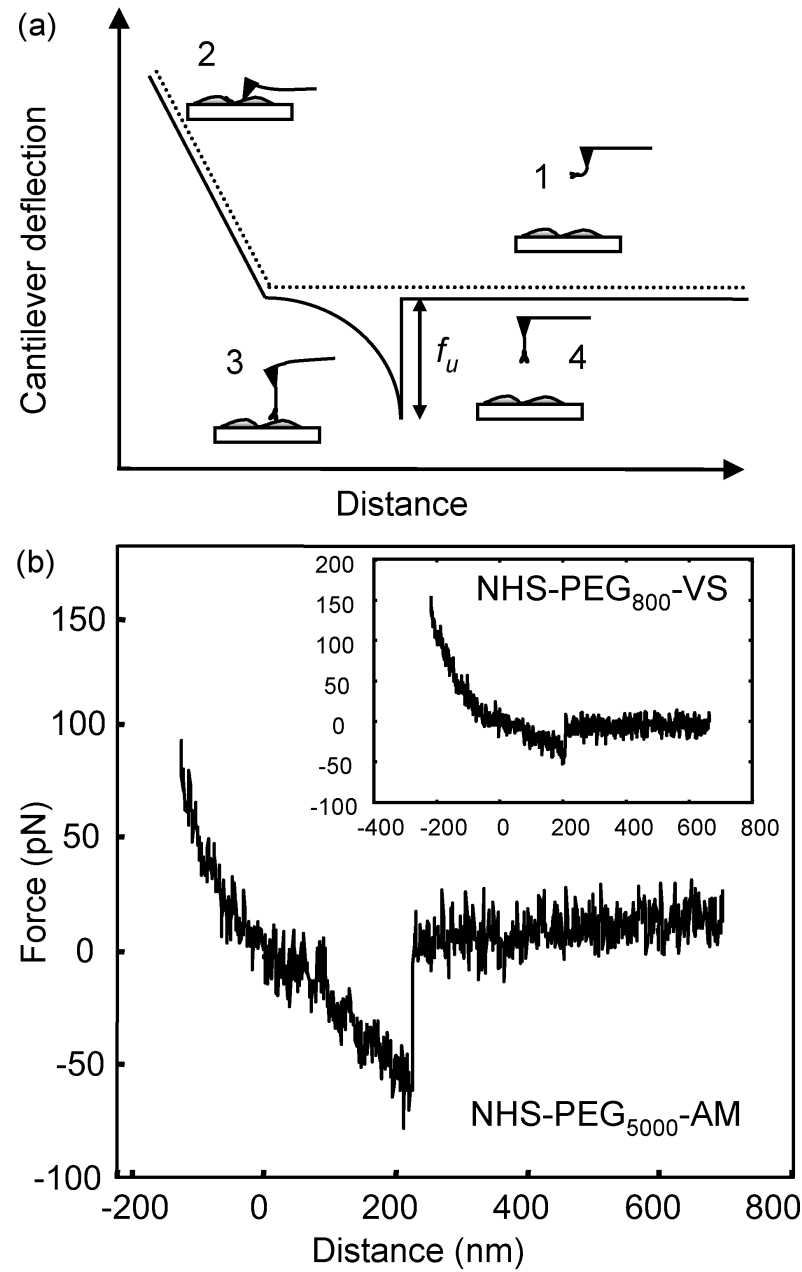

Fig. 4. Single-molecule recognition event detected with AFM. (a) Schematic representation of a force-distance cycle on live cells. The tip was moved towards the cell surface (dotted line, 1-2) and then retracted (solid line) at a constant lateral position. During tip approach, the ligand specifically binds with a receptor that leads to a force signal with a distinct shape (3) during tip retraction. The force increases until bond-rupture occurs (4) at the unbinding force $\left(f_{u}\right)$. (b) Typical force curves showing specific interactions between glucose and SGLT1 upon tip-surface retraction by using $\mathrm{PEG}_{5000}-\mathrm{AM}$ and $\mathrm{PEG}_{800}-\mathrm{VS}$ (inset) crosslinkers.

in the contact region is observed (2 to 3 ). In the case when the sugar on the tip has bound to a transporter on the cell surface, an attractive force develops (downward deflection) and the crosslinker via which the sugar is coupled to the tip will be stretched more and more with increasing tip-surface separation, leading to a force signal of distinct shape (3). Finally, the sugar will detach from the transporter ( 3 to 4 ) at a particular unbinding force, $f_{u}$ (see arrow) and the cantilever jumps back to zero deflection (4). The sweep-amplitude of the forcedistance cycles was $1000 \mathrm{~nm}$ at a $1-\mathrm{Hz}$ sweep rate. Up to 500 - 1000 force-distance cycles were performed for each location on the surface of cells and up to four locations 


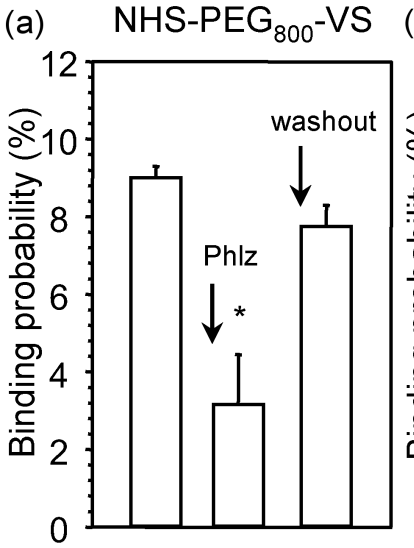

(b) NHS-PEG ${ }_{800}-\mathrm{AM}$

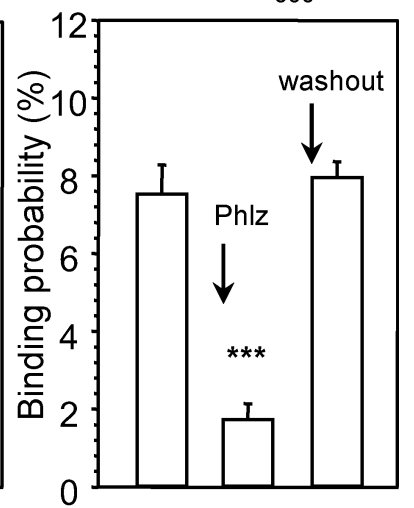

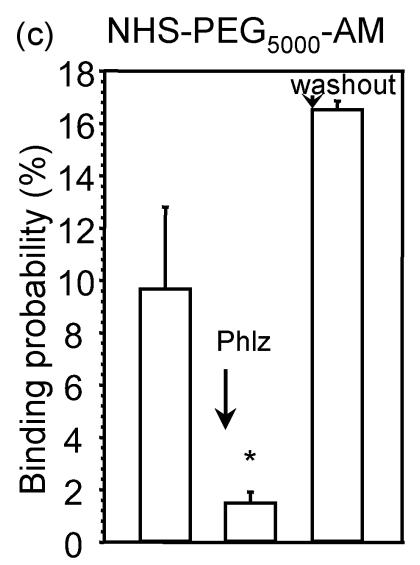

Fig. 5. Inhibition of D-glucose binding with a competitive phlorizin inhibitor. Binding probabilities of D-glucose coupled to AFM tips via (a) $\mathrm{PEG}_{800}-\mathrm{VS}$, (b) $\mathrm{PEG}_{800}-\mathrm{AM}$ and (c) PEG $5000-A M$ crosslinkers and of the block by phlorizin are shown. Values are means \pm SEM (standard error of the mean); $n=2000-4000$. Phlorizin is abbreviated by Phlz. * $=\mathrm{p}$-value $<0.05$ and $^{* * *}=\mathrm{p}$-value $<0.005$.

(different cells) for each condition, i.e., initial condition, ligand addition and washout condition.

Glucose/SGLT1 interaction was observed with all three crosslinkers. Figure 4(b) shows typical force curves (retraction) of a single molecule sugar-SGLT1 recognition event obtained by using glucose tips coupled via $\mathrm{PEG}_{5000}-\mathrm{AM}$ and $\mathrm{PEG}_{800}-\mathrm{VS}$ (inset). The specificity of the recognition of SGLT1 by glucose was confirmed by blocking experiment through the addition of phlorizin, a specific high-affinity competitive inhibitor that binds to the external membrane surface of SGLT1. The binding probability (probability of finding an unbinding event in force-distance cycles) was reduced significantly from $9.0 \pm 0.3 \%$ to $3.2 \pm 1.3 \%$ (Figure $5(\mathrm{a})$ ), from $7.5 \pm 0.8 \%$ to $1.7 \pm 0.4 \%$ (Figure $5(\mathrm{~b})$ ) and from $9.7 \pm 3.1 \%$ to $1.5 \pm 0.4 \%$ (Figure $5(\mathrm{c})$ ) for D-glucose coupled to AFM tips via $\mathrm{PEG}_{800}-\mathrm{VS}, \mathrm{PEG}_{800}-\mathrm{AM}$ and $\mathrm{PEG}_{5000}-\mathrm{AM}$, respectively. After phlorizin was subsequently washed out, the binding probability increased again (Figures 5(a)-(c)). By constructing an empirical probability density function of the unbinding forces, the maximum forces of the distribution from several experiments were found to be 40.1, $49.6 \mathrm{pN}$ and $65.7 \mathrm{pN}$ for the glucose coupled to AFM tips via $\mathrm{PEG}_{800^{-}} \mathrm{VS}, \mathrm{PEG}_{800^{-}}$ $\mathrm{AM}$ and $\mathrm{PEG}_{5000}-\mathrm{AM}$, respectively (data not shown). A student t-test analysis unequivocally revealed that the differences between $\mathrm{D}$-glucose coupled via $\mathrm{PEG}_{800}$-VS and $\mathrm{PEG}_{5000}-\mathrm{AM}$ were statistically significant (p-value $<0.005)$. The higher forces measured when D-glucose was coupled to the AFM tip via acrylamide suggest that D-glucose might be able to penetrate further into SGLT1 in this configuration and, thus, partly image the sugar transport pathway of SGLT1. In contrast and as already mentioned earlier, the vinylsulfon crosslinker contains a bulky group, most likely allowing only for superficial binding and obstructing glucose transport. Therefore, the acrylamide crosslinkers appear to be suitable crosslinkers for further investigations of the sugar transport pathway of SGLT1.

\section{CONCLUSION}

These studies demonstrate that AFM force spectroscopy is a useful method for exploring the functional dynamics of SGLT1 in live cells on a single-molecule level. Specific glucose/SGLT1 interactions were observed using 1-thio- $\beta$-D-glucose coupled to AFM tips via crosslinkers carrying vinylsulfon and acrylamide end groups. The strikingly different unbinding force values measured using these crosslinkers might arise from the differences in the accessibility of glucose to transmembrane parts of SGLT1. We, thus, hypothesize that a long, thin acrylamide-PEG crosslinker possibly allows glucose to penetrate deeper into the transporter than the other two crosslinkers. Therefore, we suggest that this crosslinker might be a promising candidate for further single-molecule studies elucidating the glucosetranslocation pathway.

\section{ACKNOWLEDGMENTS}

We are indebted to Linda Wildling for her helpful synthesis of the AFM crosslinkers. This work was supported in part by the Thailand Research Fund (T.P.) and by the National Nanotechnology Center (NANOTEC), NSTDA, Ministry of Science and Technology, Thailand, through its program of Center of Excellence Network. The Max Planck Institute of Molecular Physiology, Dortmund, Germany and the Austrian Science Foundation Project P-14549 (P.H.) are gratefully acknowledged.

\section{REFERENCES}

[1] M. A. Hediger and D. B. Rhoads, Physiol. Rev. 74, 993 (1994). 
[2] E. M. Wright, Am. J. Physiol. Renal Physiol. 280, F10-8 (2001).

[3] R. K. Crane, Rev. Physiol. Biochem. Pharmacol. 78, 99 (1977).

[4] T. Puntheeranurak, L. Wildling, H. J. Gruber, R. K. Kinne and P. Hinterdorfer, J. Cell Sci. 119, 2960 (2006).

[5] R. Lal and S. A. John, Am. J. Physiol. 266, C1 (1994).

[6] G. Binnig, C. F. Quate and C. Gerber, Phys. Rev. Lett. 56, 930 (1986).

[7] J. H. Hoh and P. K. Hansma, Trends Cell Biol. 2, 208 (1992).

[8] G. Pfister, C. M. Stroh, H. Perschinka, M. Kind, M. Knoflach, P. Hinterdorfer and G. Wick, J. Cell Sci. 118, 1587 (2005).

[9] V. Dupres, F. D. Menozzi, C. Locht, B. H. Clare, N. L. Abbott, S. Cuenot, C. Bompard, D. Raze and Y. F.
Dufrene, Nat. Methods 2, 515 (2005).

[10] E. L. Florin, V. T. Moy and H. E. Gaub, Science 264, 415 (1994).

[11] P. Hinterdorfer, W. Baumgartner, H. J. Gruber, K. Schilcher and H. Schindler, Proc. Natl. Acad. Sci. U.S.A. 93, 3477 (1996).

[12] G. U. Lee, L. A. Chrisey and R. J. Colton, Science 266, 771 (1994).

[13] S. Wielert-Badt, P. Hinterdorfer, H. J. Gruber, J. T. Lin, D. Badt, B. Wimmer, H. Schindler and R. K. Kinne, Biophys. J. 82, 2767 (2002).

[14] F. Liu, F. T. Arce, S. Ramachandran and R. Lal, J. Biol. Chem. 281, 23207 (2006).

[15] H. Kipp, S. Khoursandi, D. Scharlau and R. K. Kinne, Am. J. Physiol. Cell Physiol. 285, C737 (2003). 